\title{
Summary of 11th International Symposium on High Energy Spin Physics
}

\author{
A. D. Krisch \\ Randall Laboratory of Physics, University of Michigan \\ Ann Arbor, Michigan 48109-1120 USA
}

I greatly enjoyed attending this joint meeting of the 11th International Symposium on High Energy Spin Physics and the 8th International Symposium on Polarization Phenomena in Nuclear Physics. This SPIN ' 94 meeting provided an excellent opportunity for nuclear and high energy spin physicists to learn something from each other in one of my favorite towns, Bloomington. I will mostly stick to my job of summarizing the High Energy Spin Symposium, but I may accidentally mention a few exciting Nuclear Spin topics.

There was an excellent historical introduction to Electron Spin Physics in the talk of Professor Ternov. In 1963, along with Professor Sokolov, he discovered the Sokolov-Ternov effect of self-polarization, ${ }^{1}$ where electrons and positrons become polarized along the accelerator magnets' vertical field direction because of their different spin-up and spin-down synchrotron radiation rates. This self-polarization has recently become very important to our field. In the 1960's, self-polarization seemed a clever abstraction, which was only interesting to theorists. Now HERA and LEP, two of the world's largest electron facilities, both operate with polarized beams using the Sokolov-Ternov self-polarization effect. It was a pleasure and an honor to have Professor Ternov lecture at this Symposium.

Turning to the history of proton-proton spin effects, I will show Figure 1, which seems especially appropriate since this meeting includes both high energy and nuclear spin physicists. It displays the spin-spin correlation parameter for $90_{c m}^{\circ}$ proton-proton elastic scattering from the lowest up to the highest measured energy; ${ }^{2}$ Professor Haeberli helped me to make this compilation. When I started studying spin around 1970, most people were quite sure that there would be no spin effects at high energy. This graph certainly does not support that belief.

After these large two-spin effects were discovered at the ZGS, ${ }^{3}$ many people said, "Perhaps there are two-spin effects when the beam and the target are both polarized, but, surely there will be no one-spin effects at high energy." The talks by Professors Devlin and Pondrom ${ }^{4}$ on inclusive hyperon polarization and 
hyperon magnetic moments referred to this perturbative QCD prediction that $A$ should go to zero at high energy and high $\mathrm{P}_{\perp}$ for all hadronic reactions. Figure 2 shows the hyperon polarization plotted against transverse momentum at $12 \mathrm{GeV}$, at $400 \mathrm{GeV}$, and at $2000 \mathrm{GeV}$; this data certainly does not support the $\mathrm{A}=0$ prediction. Moreover, it seems to me that $2000 \mathrm{GeV}$ is a fairly high energy.
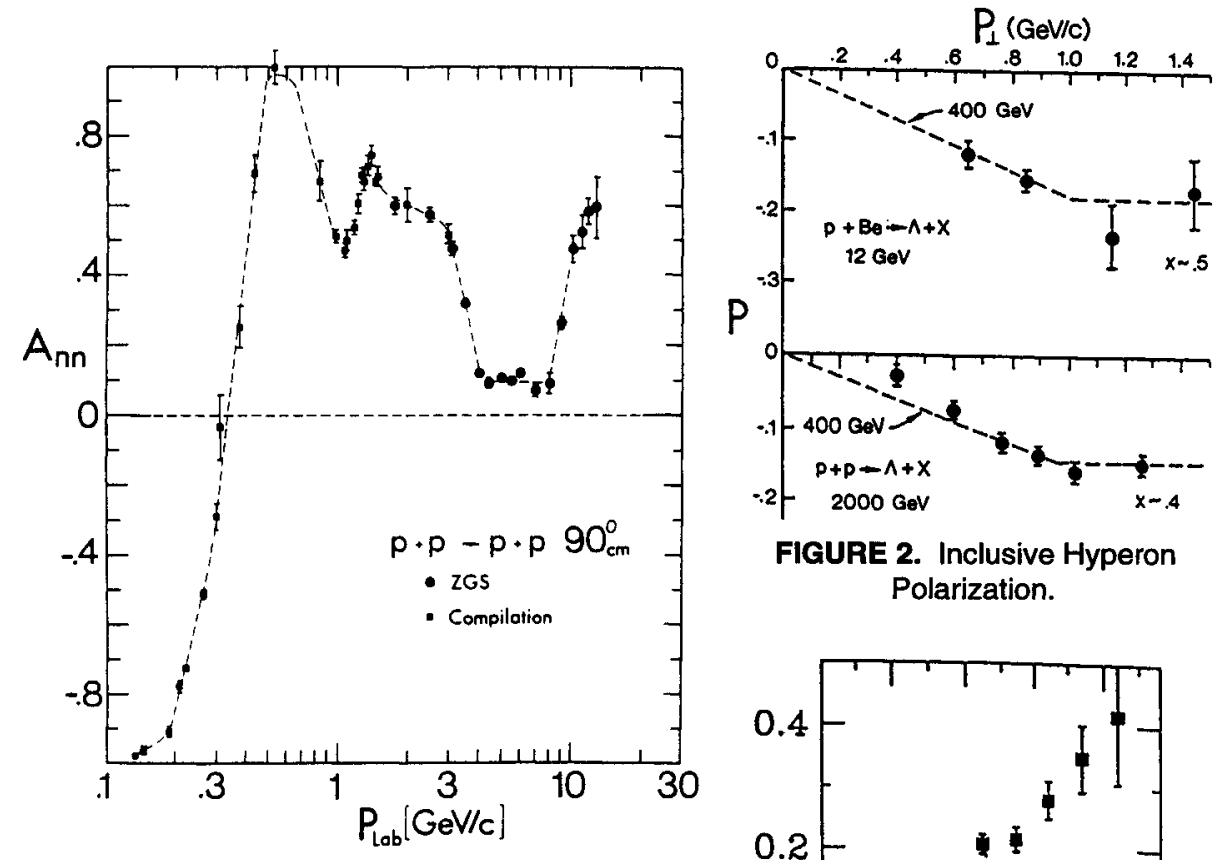

FIGURE 2. Inclusive Hyperon Polarization.

FIGURE 1. Momentum Dependence of Proton-Proton Elastic Spin-Spin Correlation Parameter at $90^{\circ}$.

Figure 3 shows some interesting results ${ }^{5}$ from E-704 at Fermilab, where they used the $200 \mathrm{GeV}$ polarized proton beam produced by hyperondecay to measure the left-right asymmetry in inclusive pion production. Notice that, at large Feynman-x, there are certainly large left-right asymmetries which should not exist, according to PQCD.

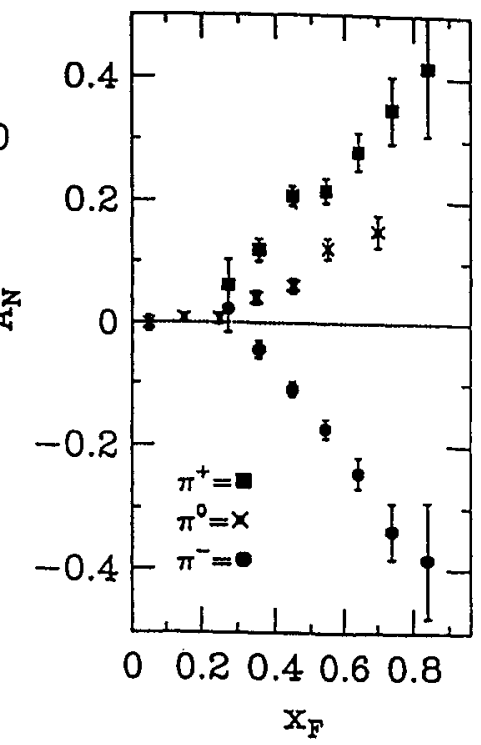

FIGURE 3. Fermilab E-704:

Left-Right Asymmetry in Inclusive Pion Production. 
Some people noted that, in these experiments, $P_{\perp}$ was only about $1 \mathrm{GeV} / \mathrm{c}$; they said that perturbative $\mathrm{QCD}$ would surely force one-spin asymmetries to go to zero at higher $P_{\perp}$. Figure 4 shows some elastic one-spin data from the AGS and CERN. The prediction of perturbative QCD for elastic scattering in this region was again that A should be zero; the AGS data ${ }^{6}$ certainly do not agree with this $A=0$ prediction at $P_{\perp}{ }^{2}=7(\mathrm{GeV} / \mathrm{c})^{2}$. Thus, the predictions of the PQCD theory of hadronic interactions do not agree with these four hadronic spin experiments. I was quite amused by the earlier quoted comment of Bjorken that perhaps theorists on Program Committees should ban spin experiments to help protect PQCD.

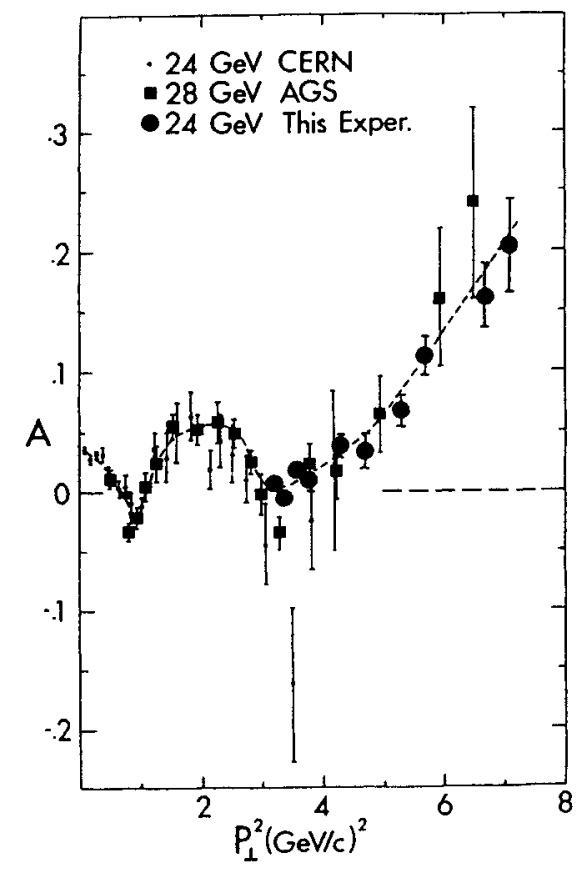

FIGURE 4. Spin Asymmetry in p-p Elastic Scattering at High $P_{\perp}$.

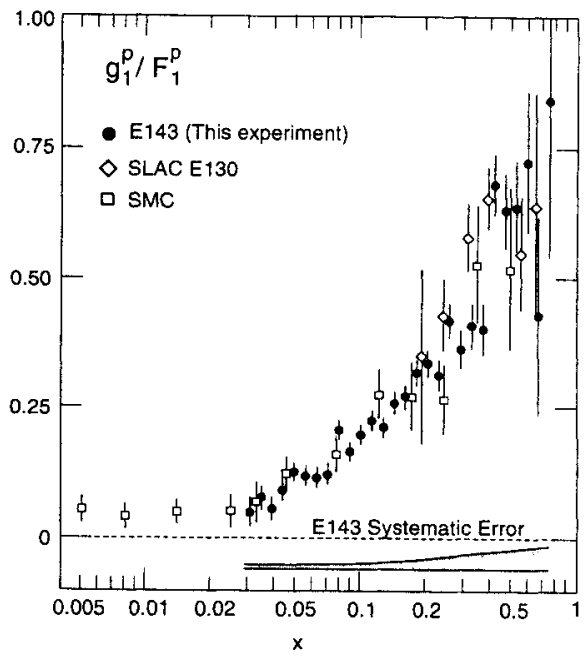

FIGURE 5. "Spin Crisis" Form Factor Ratio Plotted Against $x$.

Our most recent excitement has been the "Spin Crisis"; we heard many comments about this in the talks of Thomas, R. Voss, Windmolders, Day, Jackson, Soffer, and Anselmino. ${ }^{7}$ We heard from Professor Thomas that perhaps now there is not so much of a spin crisis. I then asked him, "What changed? the data, the theory, or the definition of 'crisis'?" Apparently, there have been some small changes in the data, but Figure 5 shows that the recent SMC data using a CERN polarized muon beam on a polarized proton target agrees rather well with the SLAC polarized electron data from experiments E-130 and E-143. Turning to the theory, perhaps there was a bit of overconfidence in the validity of some sum rules and some extrapolations to very small values of $x$, which had not been experimen- 
tally tested; now they have been tested. But probably the biggest change was in our definition of the word "crisis"; the cancellation of the SSC helped us to better understand the word crisis. In any case, the data now indicates that probably each proton does not contain three simple quarks which carry most of the proton's spin; Professor Prescott told me that the best present estimate is that the quarks and anti-quarks together carry about $1 / 3$ of the proton's spin.

There were reports about three different workshops. Professor Anderson reported on the May 1993 Workshop on Polarized Ion Sources and Polarized Gas Targets in Madison, Wisconsin; ${ }^{8}$ Professor Mori ${ }^{9}$ also reviewed this subject. Many exciting results were presented, but I will only mention Figure 6 which shows the intense atomic beam sources at Heidelberg and Wisconsin. The present source technology is very impressive, especially the high-gradient permanent magnet sextupoles of $4 \mathrm{~T} \cdot \mathrm{cm}^{-1}$. These new polarized sources work very well; they produce intensities of $4 \cdot 10^{16}$ per second with a polarization of over $80 \%$. The situation is now very different from 1970 when Hilton Glavish from New Zealand sold us the world's first commercial polarized proton source; it cost about $\$ 250,000$ and produced $6 \mu \mathrm{A}$. I am very impressed by the progress in polarized ion sources.

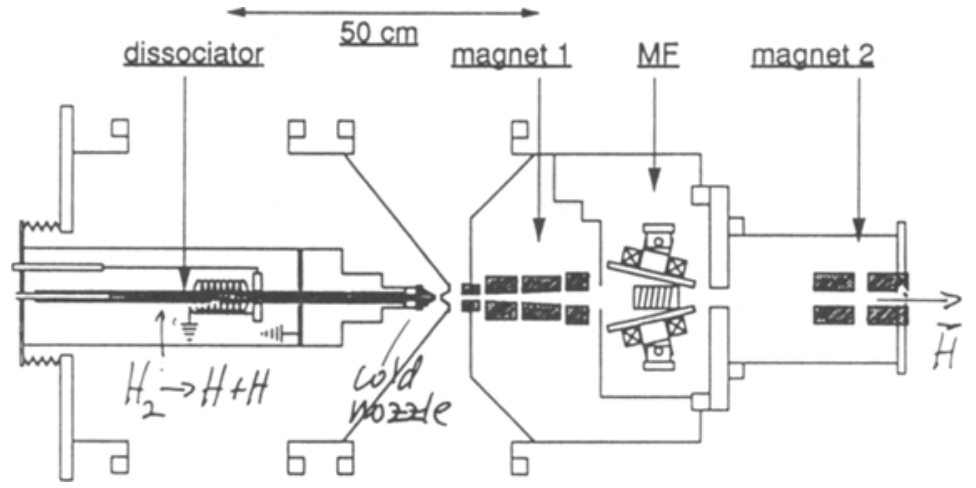

FIGURE 6. Wisconsin-Heidelberg-Marburg-Munich Source of Polarized Hydrogen Atoms.

Professor Nurushev discussed the 5th International Workshop on High Energy Spin Physics (SPIN '93), ${ }^{10}$ These Workshops, which he organizes in the odd-numbered years at Protvino, are really small symposia which allow young physicists from the former Soviet Union to hear what is happening in spin physics. Spin physics is an area of great activity in the former Soviet Union, but the currency exchange problems make travel to foreign scientific meetings very difficult. I thank Professor Nurushev for organizing these valuable workshops.

Werner Meyer reviewed the 7th Workshop on Polarized Target Materials and Techniques, ${ }^{11}$ which had 49 participants from many institutions. One high- 
light was the successful Virginia-Basel-SLAC polarized target, shown in Figure 7, which is now being used in fixed-target experiments at SLAC. Its clever arrangement of magnetic fields allows a longitudinal polarization. The target uses frozen $\mathrm{ND}_{3}$ or $\mathrm{NH}_{3}$; some recent results are shown in Figure 8: the deuteron polarization was over $30 \%$ and the proton polarization was over $70 \%$ in an intense

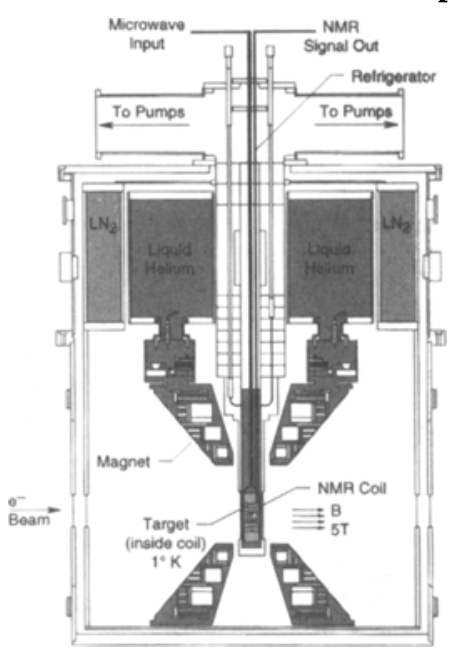

FIGURE 7. Virginia-Basel-SLAC Polarized Target. beam of several $10^{11}$ electrons per second. It is impressive that a polarized target can work so well in such extreme conditions.

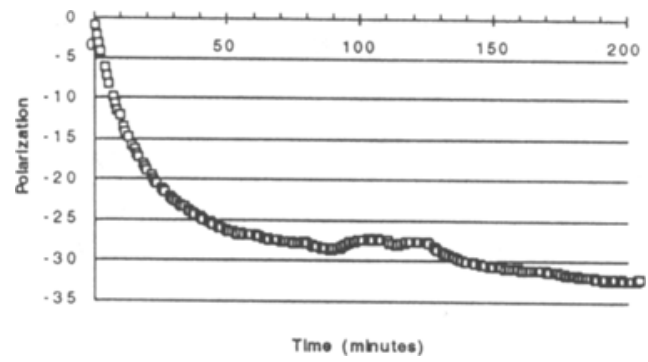

FIGURE 8. $\mathrm{ND}_{3}$ Polarization with Beam on.

One major highlight of this High Energy Spin Physics Symposium was the polarization work at the large electron facilities: SLC, HERA, and LEP. Dr. Placidi $^{12}$ gave a very nice talk on polarization at LEP; his Figure 9 shows the transverse polarization obtained at LEP in August 1993 plotted against time. Note that the polarization reached about 55\%; this clearly demonstrates the success of the

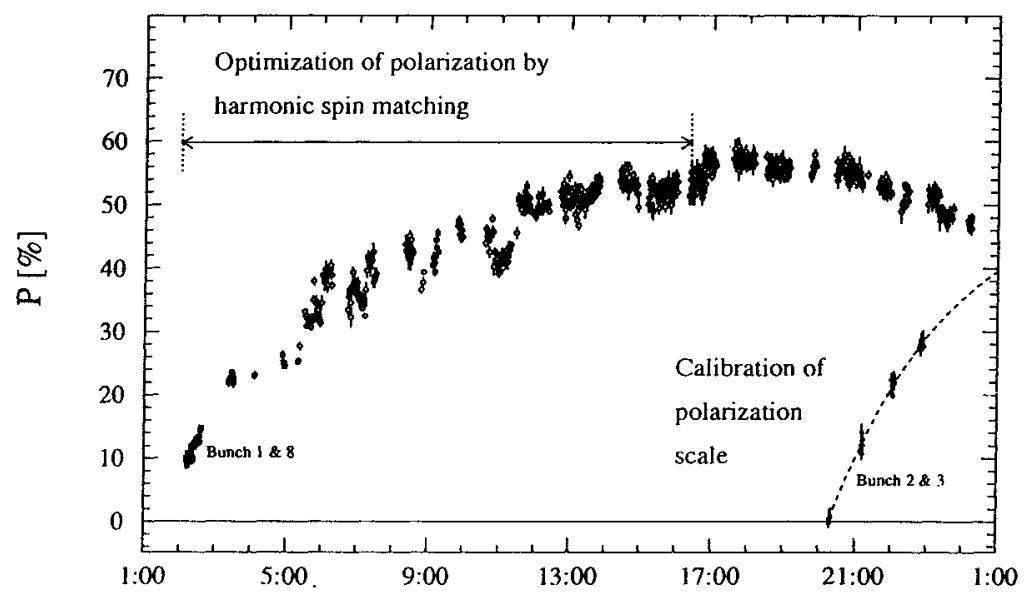

FIGURE 9. High Transverse Beam Polarization in LEP Plotted against Hours. 
Sokolov-Ternov self-polarization mechanism. ${ }^{1}$ This electron polarization was used to calibrate the LEP energy, which then provided a precise calibration of the $\mathrm{Z}$ mass. This was a rather significant contribution to high energy physics.

There has been a strong emphasis on polarization at HERA. In their talks, G. Voss, Barber, and Jackson ${ }^{13}$ each discussed various aspects of polarization at HERA. The HERA ring is shown in Figure 10 along with the H-1 experiment, the Zeus experiment, the HERMES experiment, and the spin direction at each experiment.

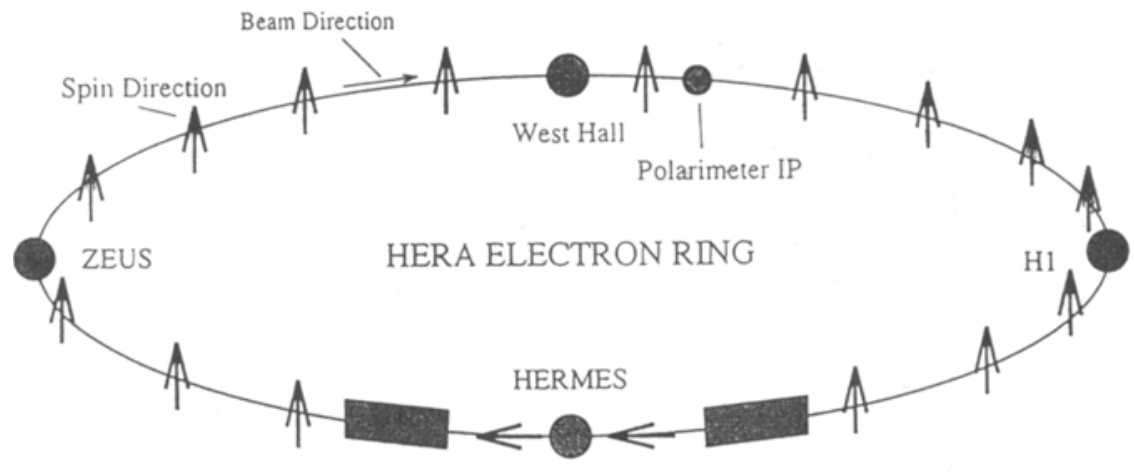

SPIN ROTATOR II

SPIN ROTATOR I

FIGURE 10. HERA Electron Ring.

I obviously cannot discuss every detail of the HERA polarization program, but I will show the HERA polarimeter in Figure 11. The incoming laser light interacts with the polarized electrons, and then the analyzer detects the scattered photons. Measuring the differences in the event rate for each spin direction gives a precise determination of the electron beam polarization. This polarimeter contains some very impressive new technology.

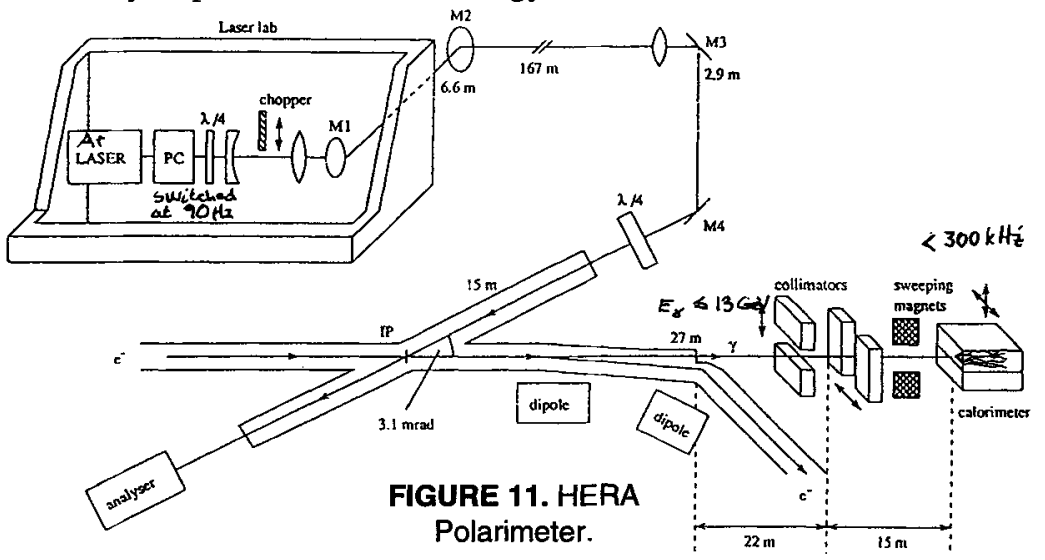


Figure 12 shows the beam polarization measured at HERA plotted against time; the transverse polarization reached about $70 \%$ and the longitudinal polarization reached about 55\% in about one hour. This was another success of the Sokolov-Ternov self-polarization mechanism. ${ }^{1}$ Also plotted is something that I named the "Soergel Limit." In a comment ${ }^{14}$ at the 1990 Bonn Spin Symposium, Professor Soergel said something like, "We decided that HERMES is approved if the HERA polarization reaches $50 \%$. If it does not reach 50\%, it is not approved." This seemed a wise thing for a Director to do; it eliminated the need for a decision and encouraged polarizers to work harder. There were about 300 witnesses to his comment, so apparently DESY decided to approve HERMES.

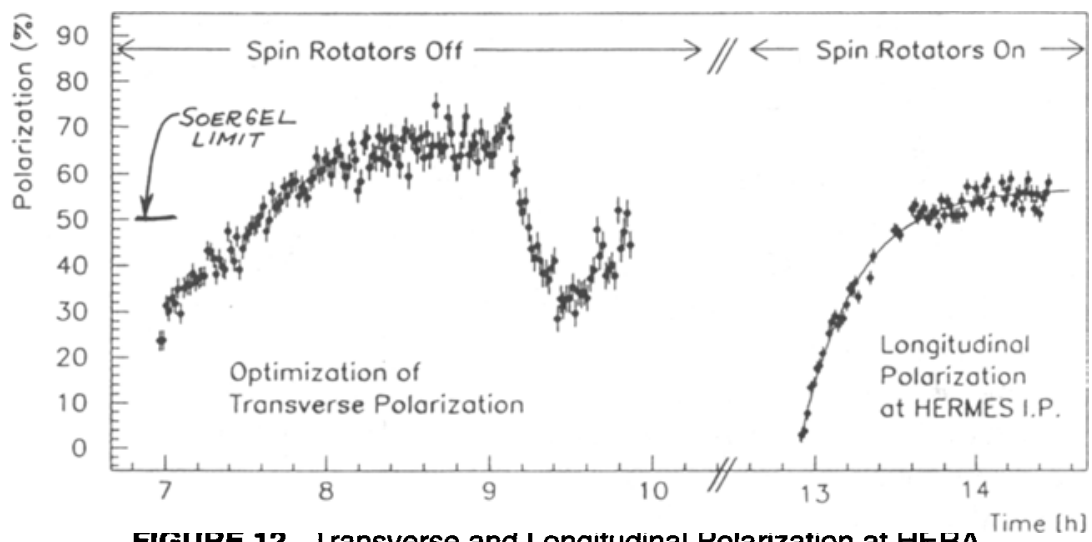

FIGURE 12. I ransverse and Longitudinal Polarization at HERA.

As shown in Figure 13, the HERMES experiment uses a "storage cell" internal target which is a bit different than a Jet. This storage cell idea is very clever. The storage cell has an incoming polarized jet, but the jet does not pass through the beam. Instead it is trapped inside the storage cell, which is open only at the ends so that the protonpolarized hydrogen atoms can only escape slowly. Therefore, most of them remain in the storage cell for some time; this increases the target thickness by a factor of 10 to 100. This storage cell is another indication that hardworking, clever, and persistent physicists can solve most problems.

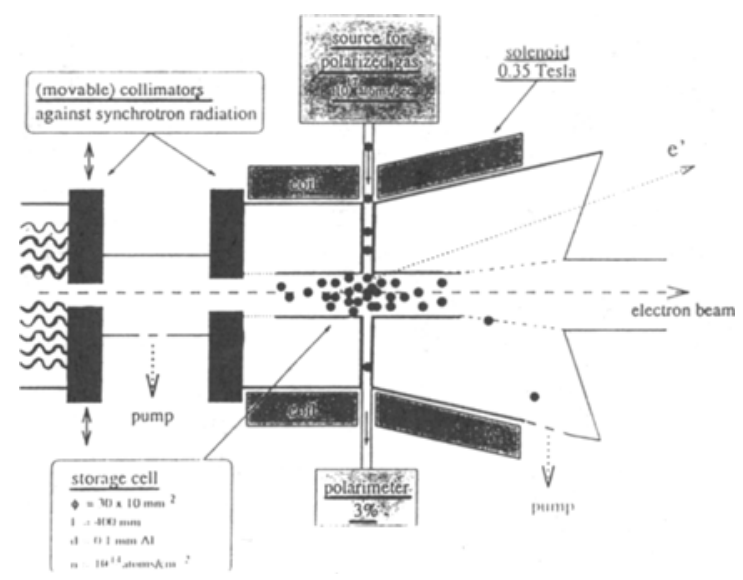

FIGURE 13. HERMES Storage Cell Target. 
I will next discuss SLAC, which now has a very nice facility called SLC. We have heard talks about SLAC and SLC from Woods, Steiner, and others. ${ }^{15}$ In recent years, SLAC, which is certainly one of the largest electron facilities in the world, has become almost exclusively a polarized electron facility. The hardware items associated with the SLAC polarized beam are shown in Figure 14. The polarized electrons are first produced in a polarized source; then one must maintain the polarization in the accumulator rings. Next the polarized electrons are accelerated in the LINAC and pass through SLC's somewhat complex non-planar arcs. Finally, one must measure the polarization near the interaction region point. So far there has been no attempt to polarize the positrons; that would be an exciting goal. Perhaps someday, we will figure out how to polarize positrons and even antiprotons.

During the past few years, the SLAC polarized source team ${ }^{15}$ made great progress with the polarized electron source, which is shown in Figure 15. The electrons are emitted by the gallium-arsenide cathode. Then the polarized laser pumps them into the proper spin-polarization state; the laser polarizes the electrons very well. In 1992, the polarization reached $40 \%$ for fixed-target running and $22 \%$ at SLC. In 1993, it reached $85 \%$ and $63 \%$. In 1994 , the polarization reached $85 \%$ for fixed-target running and $80 \%$ at SLC. These polarizations are impressive.

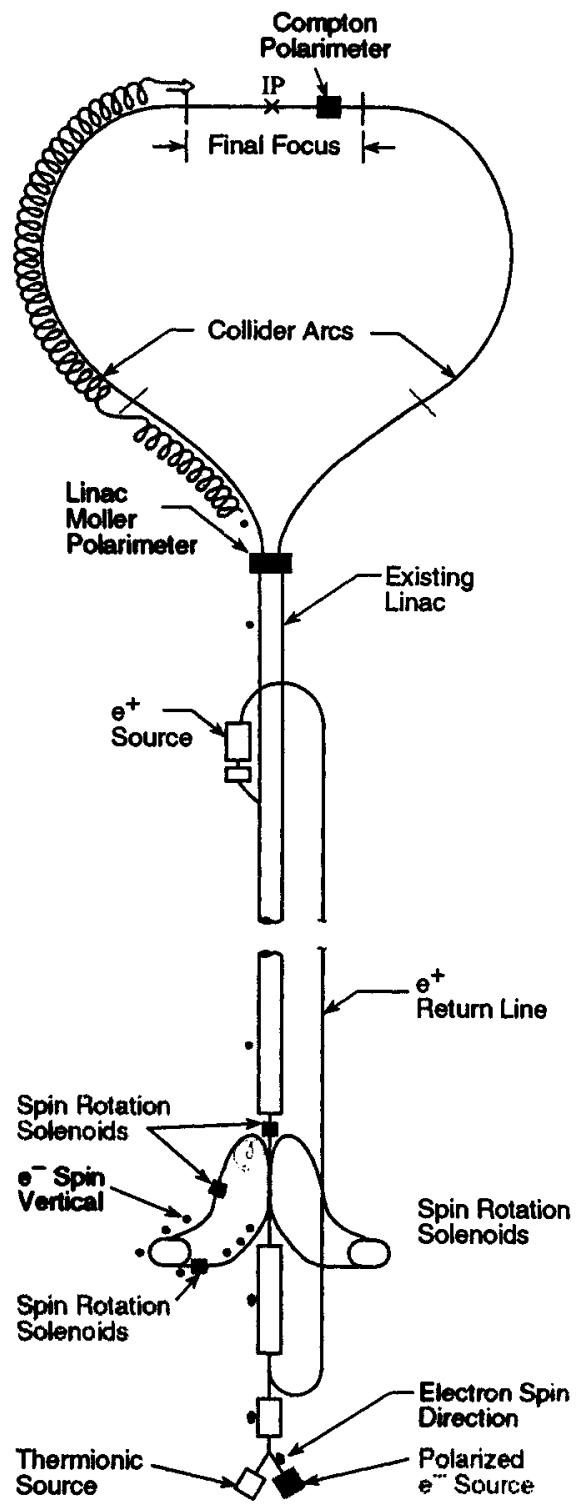

FIGURE 14. Polarization Hardware at SLAC. 


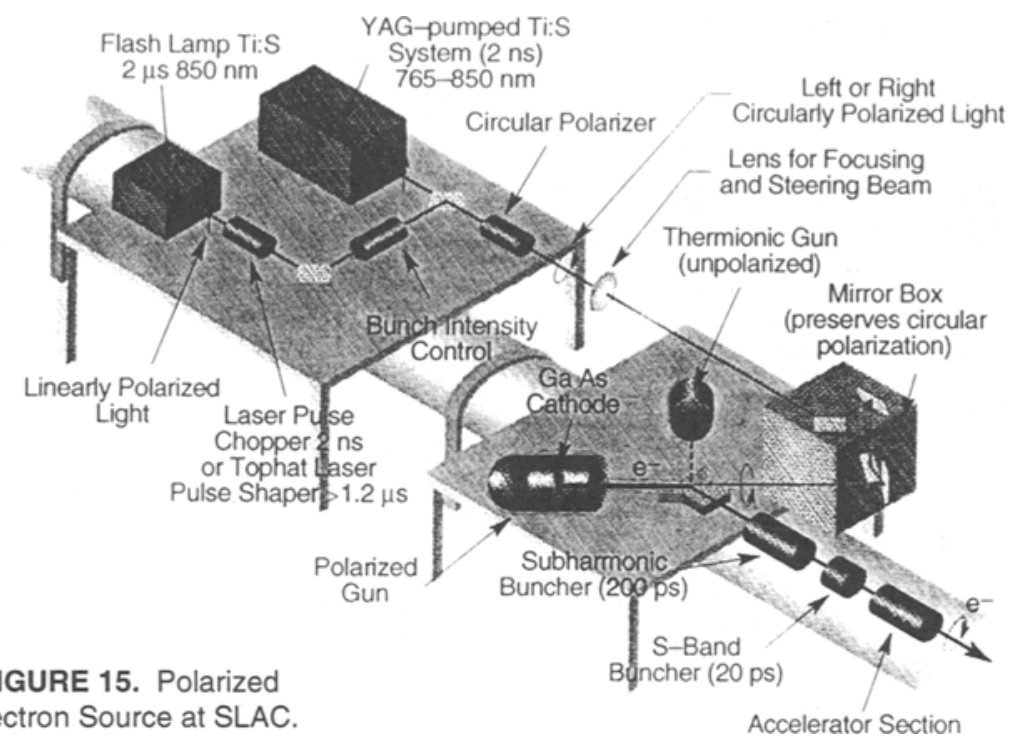

Perhaps the most significant physics result from SLAC is shown in Figure 16 , where $\sin ^{2}$ of the Weinberg angle is plotted for various experiments. The two parallel horizontal lines represent the error bars for the average of all the LEP data. The first square point on the left represents the data from SLD at SLC. All of the LEP data taken together still has a better error than the SLAC data, but only about 2.5 times better. Note that there may be some difference between the SLAC and LEP results. Using my earlier definition, I am not yet ready to call this difference a crisis, but it certainly seems interesting. Perhaps in the future, we should use the word "crisis" more sparingly and use "interesting" more often.

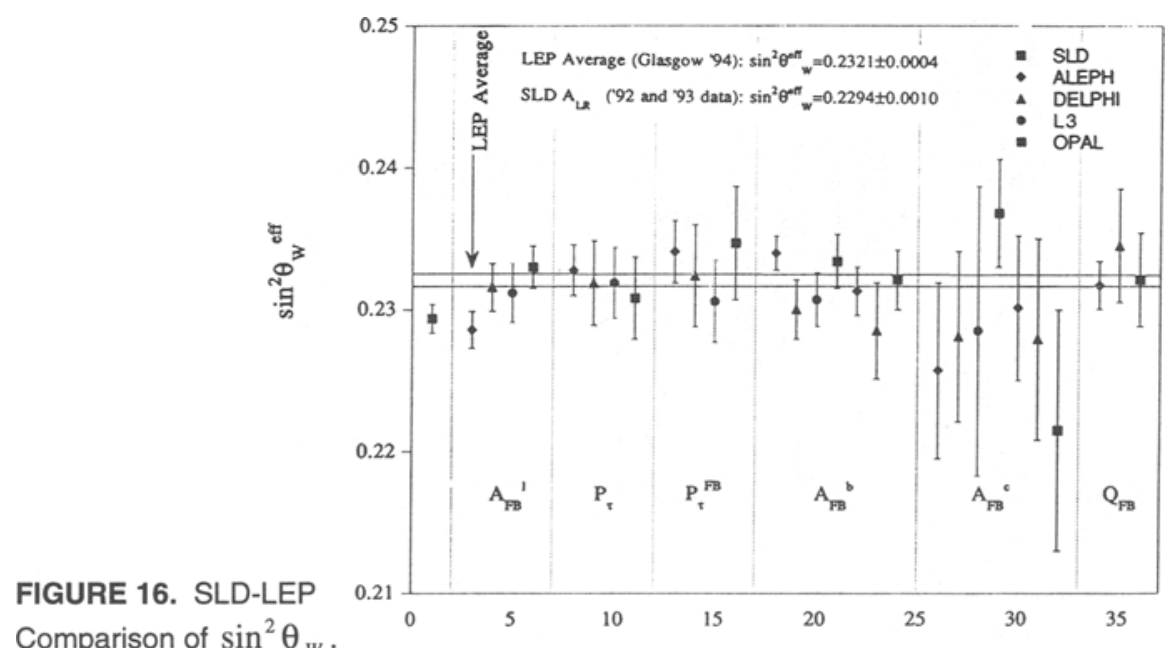


I will now discuss the proton facilities in slightly more detail; Professor Heller asked me to do this because Professor Ado was unable to give the requested lecture on polarized beams at Fermilab and UNK. I will begin with the development and testing of Siberian snakes. The Siberian snake is an extraordinarily clever idea which was invented by Derbenev and Kondratenko;16 moreover, it works.

The Siberian snake was first tested at the IUCF Cooler Ring, which is shown in Figure 17; to some people it looks like an accelerator, but we Siberian snake people think of it as an

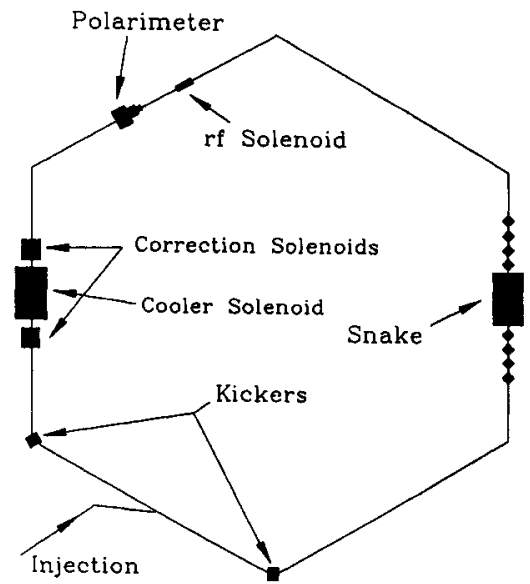

FIGURE 17. Siberian Snake at the IUCF Cooler Ring. experiment. ${ }^{17}$ Polarized protons are injected into the Cooler Ring from the IUCF cyclotrons. The kicker magnets are sometimes used for injection, and sometimes used as rf dipoles. Notice the Siberian snake, the polarimeter, the rf solenoid, and the cooling magnets which we also use to create imperfection magnetic fields for our experiments. Thus, we are using many existing hardware items for extra jobs.

The Siberian snake itself 17 is shown in Figure 18; its heart is an aging and temperamental superconducting solenoid of two Tesla-meters. The four skew and four normal quadrupoles do nothing to the spin, but they correct the solenoid's rather strong focusing and beam twisting of the 100 or $200 \mathrm{MeV}$ beam. On each turn around the ring, the snake rotates the spin by $180^{\circ}$. This makes any depolarizing effects, that may occur during one turn around the ring, cancel themselves during the next turn. The snake forces all the problems to cancel themselves; it is a very clever idea.

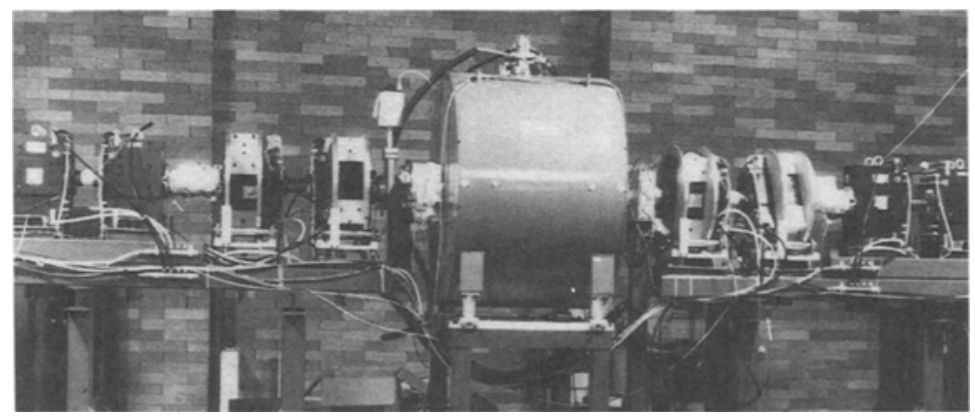

FIGURE 18. Siberian Snake at the Cooler Ring. 
We have tested many different aspects of Siberian snakes. Recently we accelerated of polarized protons from 95 to $140 \mathrm{MeV}$ through the $\mathrm{G} \gamma$ imperfection depolarizing resonance at 108 MeV. ${ }^{18}$ The measured polarization is plotted against the imperfection field integral in Figure 19; the circles show the polarization with a partial Siberian snake turned on, while the squares show the polarization with no snake. Clearly, with no snake, the polarization drops when the imperfection field is large; with the snake on, there is no depolarization. Thus the Siberian snake overcomes the depolarization during acceleration through an imperfection depolarizing resonance.

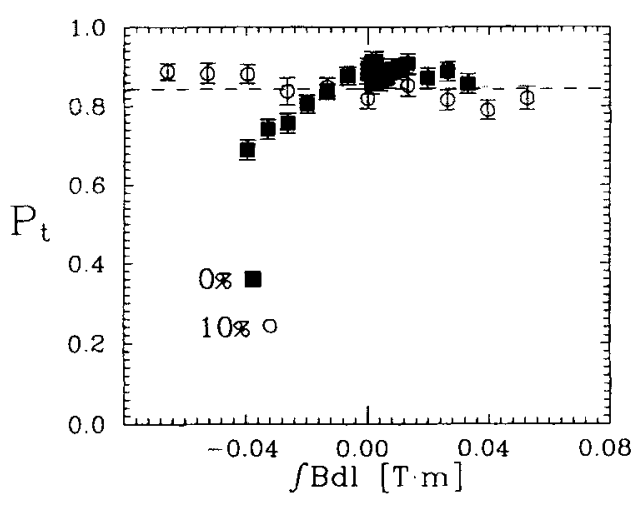

FIGURE 19. Acceleration through a Depolarizing Resonance at $\mathrm{G} \gamma \mathbf{=} \mathbf{2}$.

Since the 1992 Nagoya SPIN Symposium, we studied the adiabatic turn-on of a Siberian snake at an energy where the spin tune $\mathrm{G} \gamma$ is a half integer; this occurs at $370 \mathrm{MeV}$ at the IUCF Cooler Ring. Professor Courant had proposed that adiabatic turn-on should allow switching from one type of polarization correction to another during an acceleration cycle. The data ${ }^{19}$ demonstrated that, within our measured precision of $2 \%$, there was no polarization loss when the snake was turned on and off at these magic energies. At other energies, there is some depolarization during adiabatic turn-on.

Several speakers stressed the importance of flipping the spin of a stored polarized beam from up to down to discriminate against systematic errors. Dr. Phelps from Michigan has been much involved with our recent spin-flipping studies using an RF solenoid. The studies ${ }^{20}$ showed that, with very careful tuning, there was no polarization loss within our error of $\pm 0.05 \%$. This suggests that one can flip the spin perhaps 100 to 1,000 times without a significant loss of polarization. This is very important for reducing systematic errors. Professor Cameron seems to support further spin-flipping studies because there are several approved experiments at the IUCF Cooler Ring that need this capability.

Several high energy proton accelerators may use Siberian snakes because Professor Ternov's self-polarization formula ${ }^{1}$ does not work very well for most proton accelerators. At a Coral Gables meeting, Kent Terwilliger, R.R. Wilson, and I once calculated that self-polarization would work rather well for proton storage rings at about $70 \mathrm{TeV}$. However, until Congress funds a $70 \mathrm{TeV}$ SSC, we proton polarizers must either correct each resonance or use Siberian snakes. 
Professor Vigdor ${ }^{21}$ gave a very nice talk about Indiana's proposed Light Ion Spin Synchrotron (LISS) of approximately $20 \mathrm{GeV}$ which would use Siberian snakes to overcome the depolarizing resonances. This proposal seems rather natural since Indiana is quite familiar with Siberian snakes. LISS is shown in Figure 20; some of its parameters are: stored polarized beams of about $20 \mathrm{GeV}$ for protons and other light ions, a very high luminosity of $10^{33} \mathrm{~cm}^{-2} \mathrm{~s}^{-1}$, and long straight sections. LISS could provide a very exciting spin physics program with strong components in both High Energy Physics and Nuclear Physics. This successful SPIN '94 Meeting at IUCF should help to focus the scientific program for LISS.

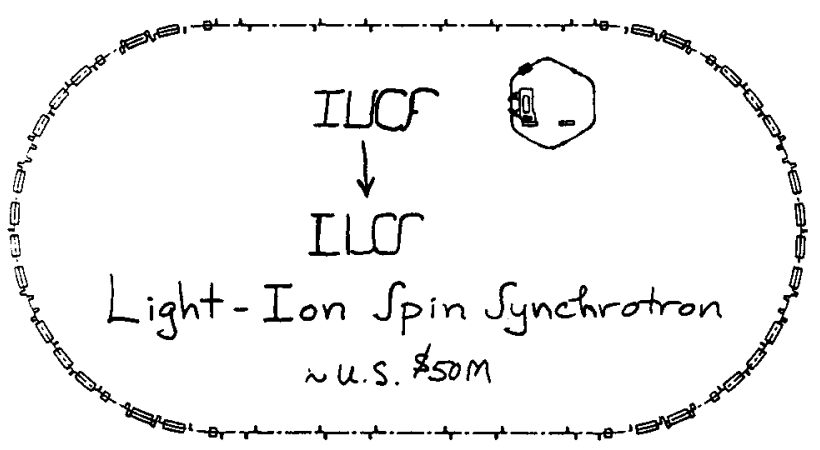

FIGURE 20. IUCF Light lon Spin Synchrotron.

For six years, the Michigan-IUCF Siberian snake experiments had no competition anywhere in the world. This is no longer true; now there is another Siberian snake in the AGS which Huang and Roser $^{22}$ discussed. Figure 21 shows a photograph of this partial warm Siberian snake; it is a rather large powerconsuming solenoid because it is quite difficult to ramp superconducting solenoids. The partial Siberian snake is rampable along with the AGS energy; it can operate as a $5 \%$ partial snake up to about $25 \mathrm{GeV}$.

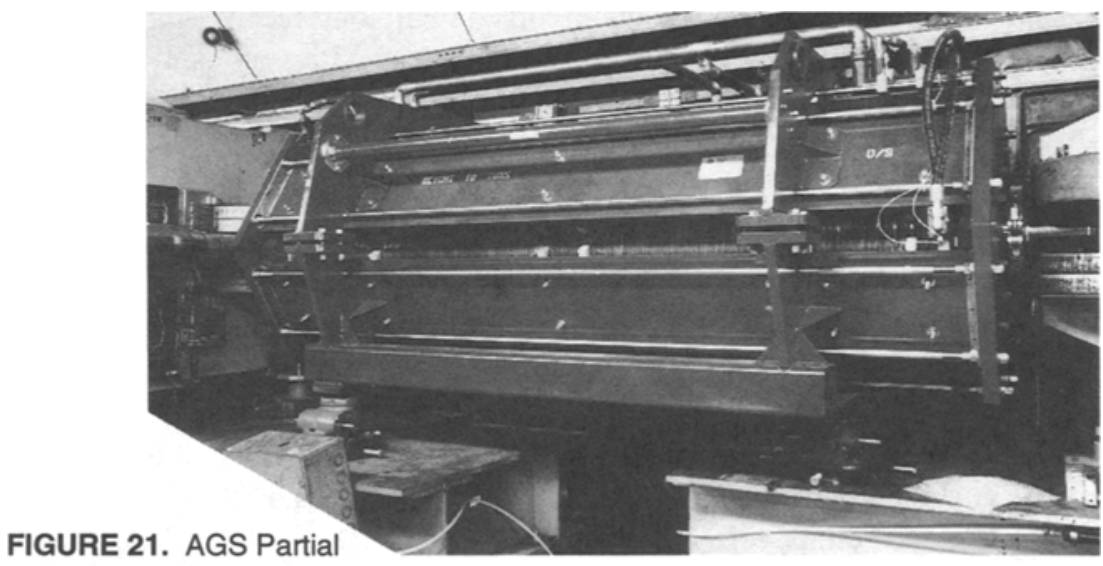
Siberian Snake. 
Some results ${ }^{22}$ from the April 1994 AGS polarized beam run are shown in Figure 22. The upper graph shows spin-flipping with the partial Siberian snake. The lower graph plots the AGS vertical polarization against $G \gamma$, which is the spin tune. The maximum $\mathrm{G} \gamma$ of about 22.5 corresponds to about $11 \mathrm{GeV}$. The snake was only a partial snake, which should overcome the imperfection depolarizing resonances but not the three intrinsic depolarizing resonances; note the significant polarization loss at each intrinsic resonance. However, the partial snake did overcome the many imperfection resonances with no observable depolarization; thus, the complex system of 96 correction dipoles ${ }^{23}$ was not needed to painfully correct them. The AGS pulsed quadrupoles were not used because their large $20 \mathrm{MW}$ power supplies had no maintenance for six years; they should allow a higher polarization in the next AGS polarized beam run.

The ability to accelerate polarized protons in the AGS, without weeks of tune-up time, may revive the AGS polarized beam physics program; moreover, it could provide polarized protons for RHIC. Dr. Makdisi24 reviewed the RHIC polarized beam program. His Figure 23 shows an overview of the polarization hardware; note the AGS's partial Siberian snake, its pulsed quadrupoles, its polarimeters, and the four full Siberian snakes and two polarimeters in RHIC, which also has eight spin rotators for the STAR and PHENIX detectors. RHIC could be the world's highest energy proton-proton collider; moreover, with Siberian snakes, both proton beams could be polarized.
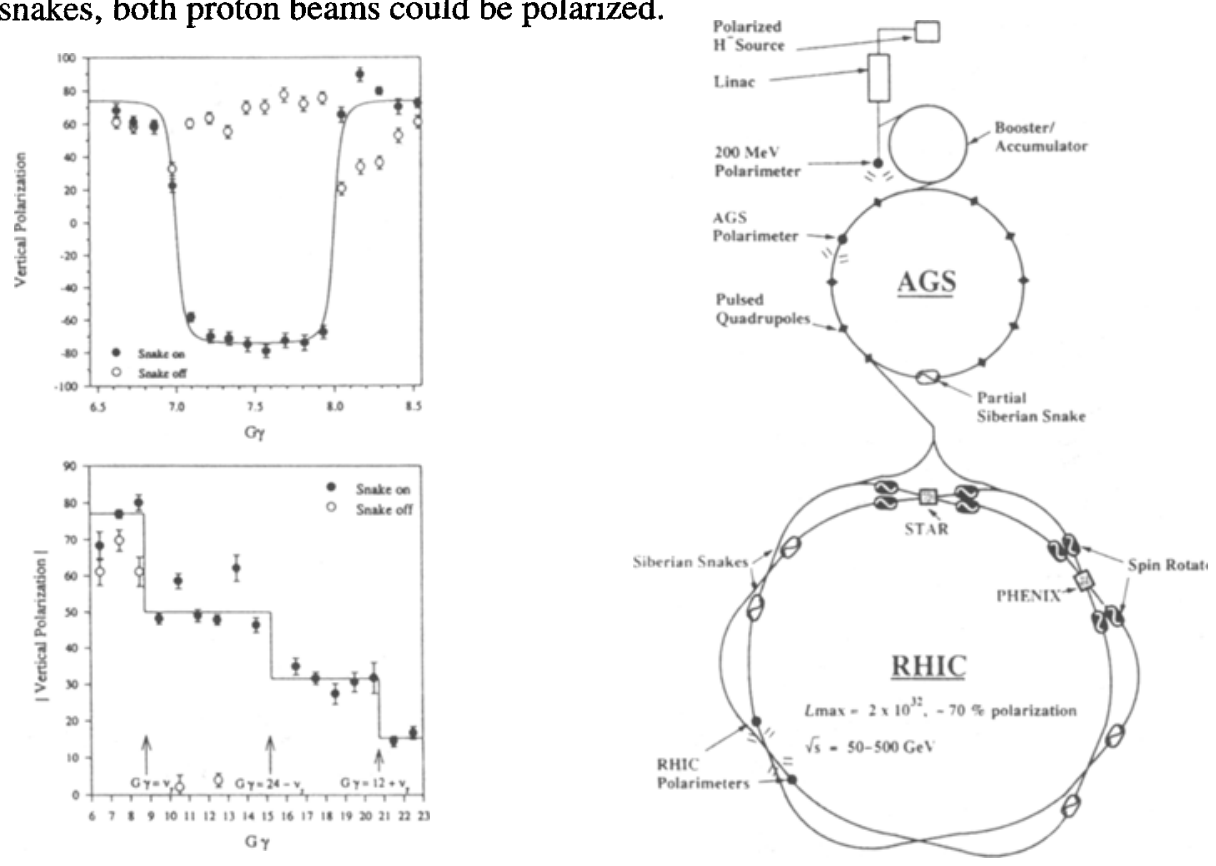

FIGURE 22. AGS Partial Snake Data.

FIGURE 23. Polarized Protons at RHIC. 
I hope that they accelerate polarized beams at RHIC; it could provide an excellent program of spin physics. The main goal of the first Workshop on High Energy Spin Physics, held in Ann Arbor in 1977, was to accelerate polarized protons in ISABEL. Now, ISABEL is long gone; its successor, SSC, is also gone. However, one may still get polarized protons in the old ISABEL tunnel. Spin physicists may not be fast, but we are persistent.

Fermilab has also shown some interest in polarized protons. In 1991 Fermilab first commissioned the SPIN Collaboration to do detailed studies of how to accelerate polarized protons in the various rings at Fermilab. Figure 24 shows the first page of the 1 August 1994 Polarized Tevatron Progress Report, ${ }^{25}$ which includes detailed drawings, budgets, and schedules on how to accelerate polarized protons in both the Main Injector and the Tevatron. Figure 25 shows the new polarized hardware required in the various Fermilab rings and injectors. Two possibilities are being considered for the polarized source: an atomic beam-type polarized source (ABS) and an optically pumped polarized ion source (OPPIS). There is a competition with equal funding going to the ABS team at IUCF and the OPPIS team at TRIUMF. Both teams have made good progress; whoever develops the best source may get to build a polarized source for Fermilab.

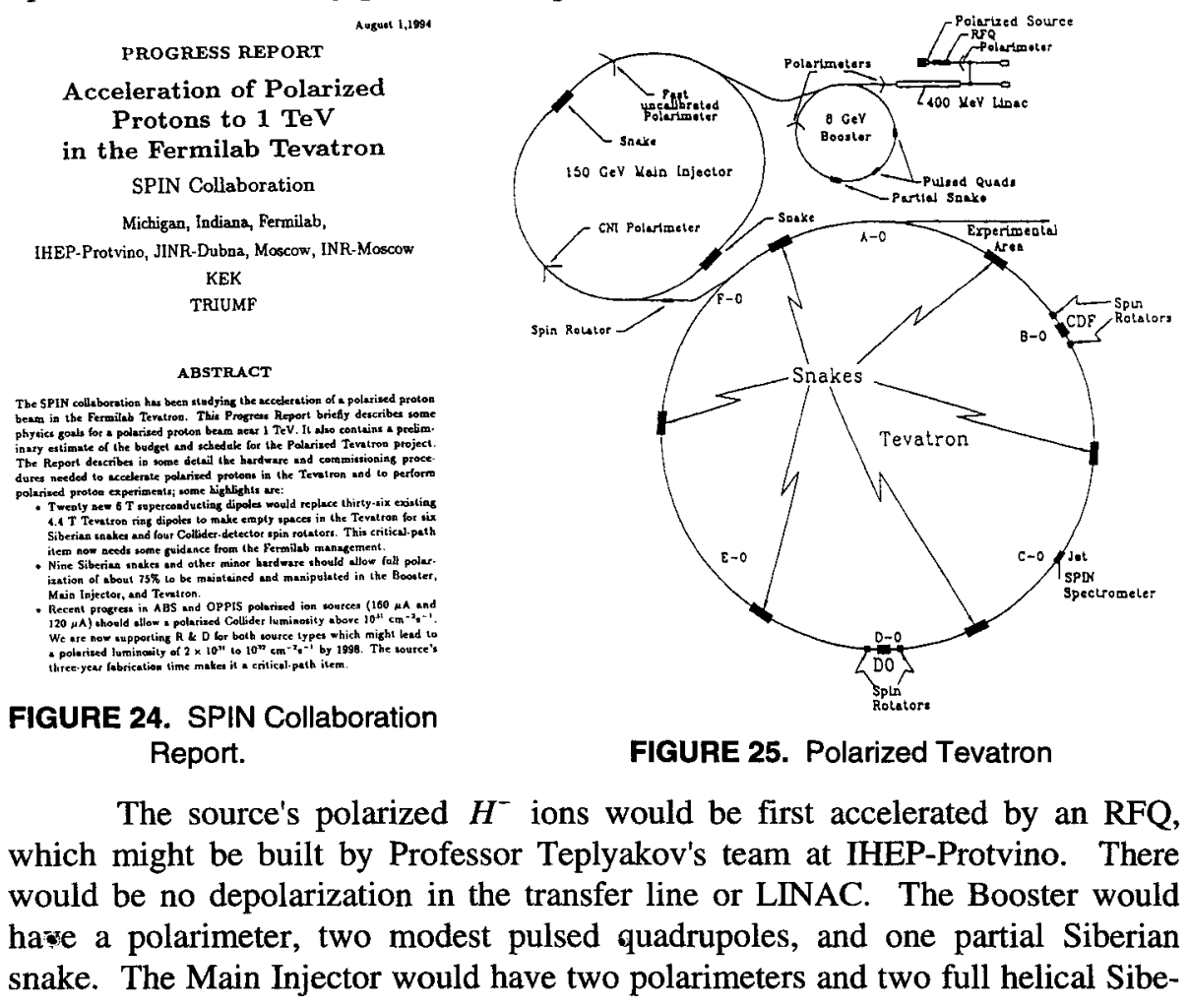


rian snakes. Professor Ado and Dr. Ludmirsky at IHEP-Protvino built a $10 \%$ scale model of a Main Injector helical dipole snake; this working model was successfully tested at both Protvino and Michigan.

There might be two fixed-target experimental areas for spin physics. In the $120 \mathrm{GeV}$ extracted beam area, there could be an experimental program using the Michigan solid polarized target which has a polarization of over $90 \%$ in a $10^{11} \mathrm{~s}^{-1}$ beam intensity. The $\mathrm{C} 0$ internal target area may have a Mark-III polarized jet, similar to the Mark-II jet for UNK (see Figure 30).

The most complex part of the Polarized Tevatron project came from the discovery that there were no places to put the Tevatron Siberian snakes with the proper symmetry defined by Professors Derbenev and Courant; I will not discuss this symmetry in detail. In any case, we decided to make spaces for the six $\mathrm{Te}-$ vatron snakes by removing at each snake point four existing 6-m-long 4.4 Tesla dipoles. We could then install, in the resulting 24-m-long space, two 8.8-m-long 6 Tesla dipoles; this would easily create a space for one 5-m-long Siberian snake. These 6 Tesla dipoles might be slightly modified HERA dipoles, UNK-2 dipoles, or new Fermilab-designed dipoles. We are now interacting with many high-field dipole people who are usually outside the spin business.

I want to stress that this project is not yet approved. Fermilab has been funding these studies and R\&D, but they certainly have not approved the required $\$ 25$ to $\$ 30$ million; we expect a decision by mid-1995. We are developing a schedule so that the Polarized Tevatron installation, which requires removing 36 existing Tevatron ring magnets, could occur during the Main Injector installation now scheduled for Fall 1997.

While preparing an EOI, Dr. Stanfield asked us to interact with the CDF and D0 people about this Polarized Tevatron project. We had several meetings, which at first were slightly painful because the colliderdetector people and the polarized beam people have not talked much to each other for the last few decades. However, a good idea came out of this "painful" interaction. During a Polarized Tevatron meeting, Professor Weerts from Michigan State University showed Figure 26, where the inclusive cross-section for jet production is plotted against transverse energy. I had seen this graph

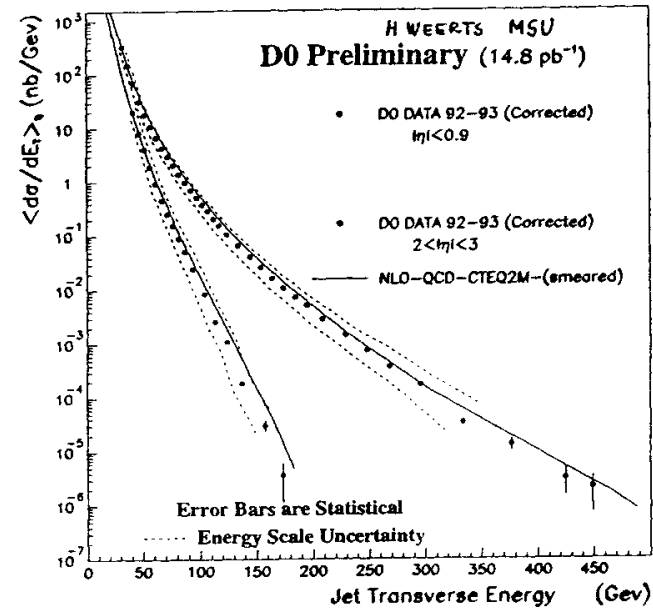

FIGURE 26. Inclusive Jet Cross Section. 
at many seminars, but I never before thought of it as being related to polarization. I suddenly saw that at a huge transverse energy (or $P_{\perp}$ ) of 100,200 , or $300 \mathrm{GeV}$, there is still a very high event rate. I remembered that perturbative $Q C D$ predicted that A must eventually be zero, but essentially ignored all existing spin experiments because they were not at "a high enough energy or high enough $P_{\perp}$ ". However, Figure 26 shows high-event-rate data at $\sqrt{s}$ of almost $2 \mathrm{TeV}$ and $\mathrm{P}_{\perp}$ of 100 or $200 \mathrm{GeV} / \mathrm{c}$. These inclusive Jets could easily test with great precision the perturbative QCD prediction that A must be zero. Weerts said that high precision measurements at $P_{\perp}=100 \mathrm{GeV} / \mathrm{c}$ can be made in a few hours. If $A$ is not zero at $\sqrt{s}=2 \mathrm{TeV}$ and $\mathrm{P}_{\perp}=100 \mathrm{GeV} / \mathrm{c}$, then it may be difficult to say where PQCD is useful.

The last proton accelerator that I will discuss is UNK, which is now being built at IHEP in Protvino, Russia; IHEP is sometimes called Serpukhov, which is a city ten miles away. IHEP's major activity now is to get the UNK-1 accelerator built and to get the NEPTUN and NEPTUN-A polarization experiments running as soon as possible. This huge new facility is shown in Figure 27. There is also an informal proposal to develop a $70 \mathrm{GeV}$ polarized proton beam at U-70 by Professor Ado and his student, Anferov, who recently proposed making spaces for the snakes in the existing U-70 ring by installing some higher field conventional magnets.

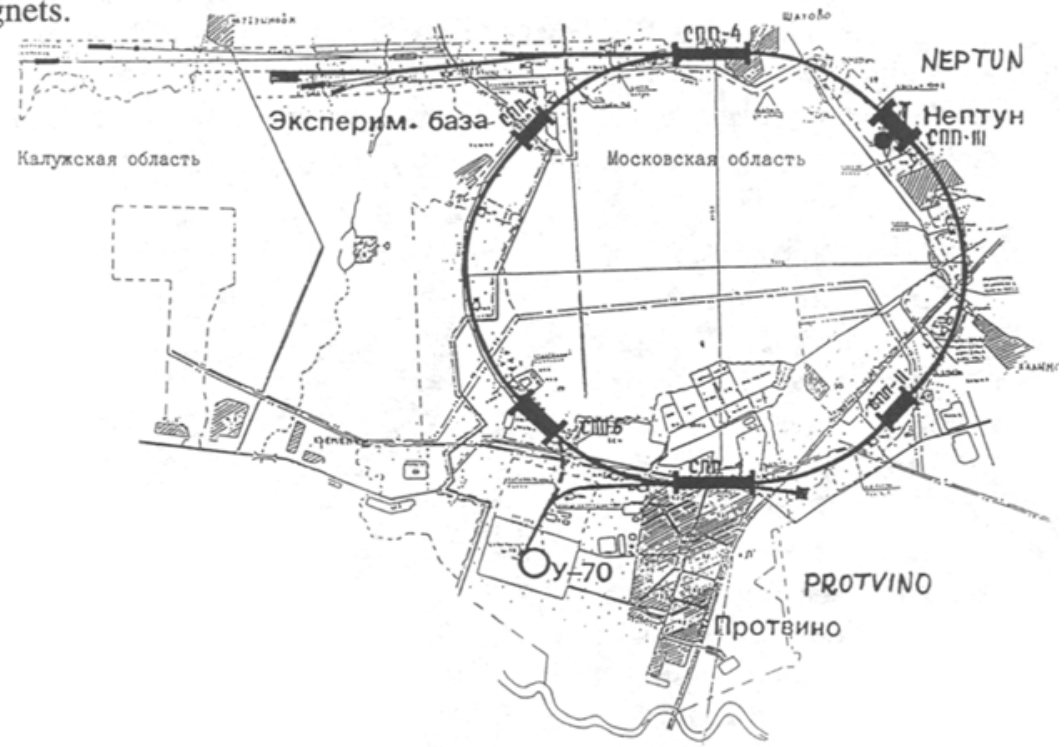

FIGURE 27. UNK and U-70 in Protvino, Russia.

In March 1994, IHEP successfully extracted a beam from the $70 \mathrm{GeV}$ accelerator U-70 and transported it through the $2.7 \mathrm{~km}$ transfer line to the UNK tunnel. ${ }^{26}$ The extraction and transfer efficiency was above $90 \%$. When all of its 
magnets are installed, the UNK tunnel will look like this transfer line, which uses UNK-1's standard 6-m-long warm dipoles, its quadrupoles, and its standard vacuum system. Figure 28 shows the transfer line. They have not yet started installing the UNK-1 magnets in the main 21-km UNK tunnel. Although about 1500 of the 2200 dipoles and all of the 500 quadrupoles are already on site, they are waiting for the tunnel air conditioning before starting to install magnets.

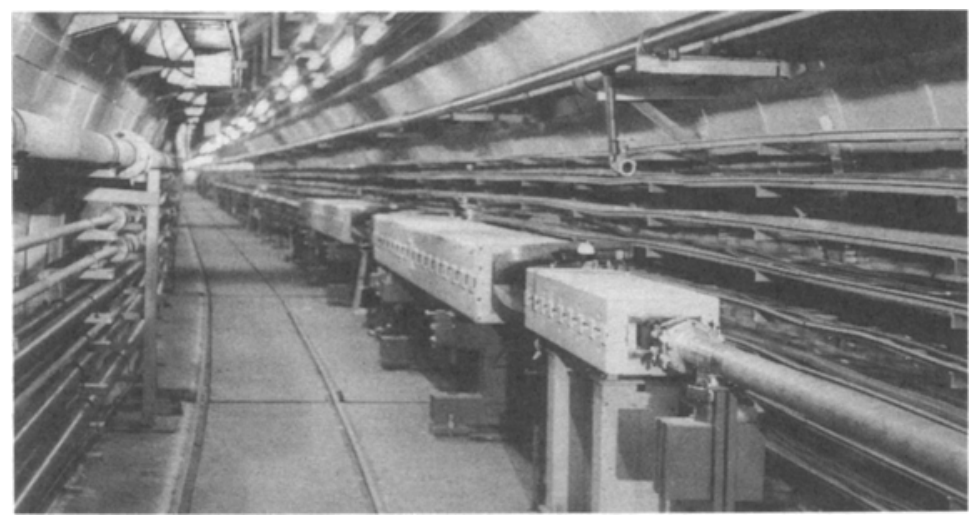

FIGURE 28. Transfer Line from the U-70 Accelerator into the UNK Tunnel.

Building UNK in these complex times in Russia has been a great challenge. However, UNK still progresses at a fairly good rate, and there is good progress in some areas. Apparently it was decided to concentrate all efforts during the next few years on finishing and then operating the 400 to $600 \mathrm{GeV}$ UNK-1 accelerator and the polarized internal jet target experiments NEPTUN and NEPTUN-A. NEPTUN is led by Professor Solovianov, and I lead NEPTUN-A. The large SS-3 underground hall, shown in Figure 29, is essentially finished; it

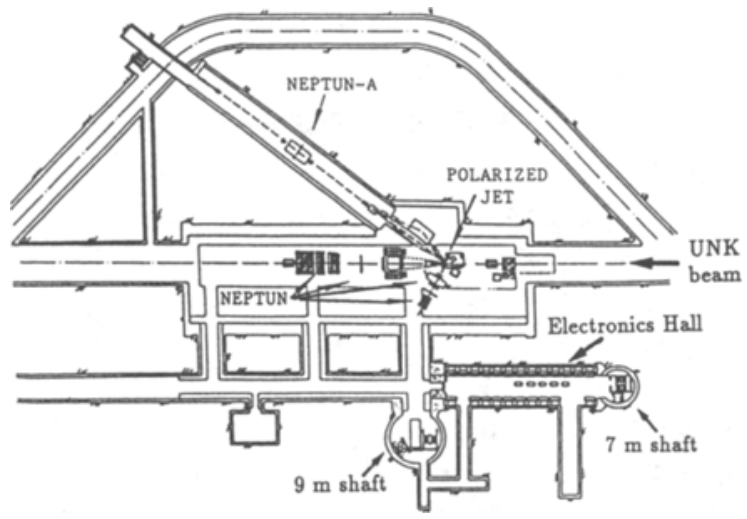

FIGURE 29. NEPTUN and NEPTUN-A Internal Polarized Jet Experiments. 
was dedicated last fall. The Mark-II polarized jet target and the NEPTUN and NEPTUN-A spectrometers are shown along with the underground electronics hall. There may also be a second upstream jet target built by Professor Pilipenko at Dubna with several smaller spectrometers. Thus, for several years, the $21 \mathrm{~km}$ circumference UNK complex may be completely devoted to polarization experiments until the 3 $\mathrm{TeV}$ superconducting UNK-2 ring operates.

Figure 30 shows the ultracold spin-polarized atomic hydrogen jet $^{27}$ which is being built at Michigan; Dr. Luppov is heading this effort. This jet uses the ultra-cold technique which was discussed at several of the sessions. We plan to take this Mark-II spin-polarized proton jet to Protvino and use it as the internal target for the NEPTUN and NEPTUN-A experiments, which will measure $A$ in $400 \mathrm{GeV}$ protonproton elastic and inelastic collisions. Our goal luminosity with this jet and UNK's $10^{19} \mathrm{~s}^{-1}$ circulating protons is $10^{32} \mathrm{~s}^{-1} \mathrm{~cm}^{-2}$.

That ends my scientific talk. I would now like to make a few comments on behalf of the International High Energy Spin Physics Committee. I would first like to

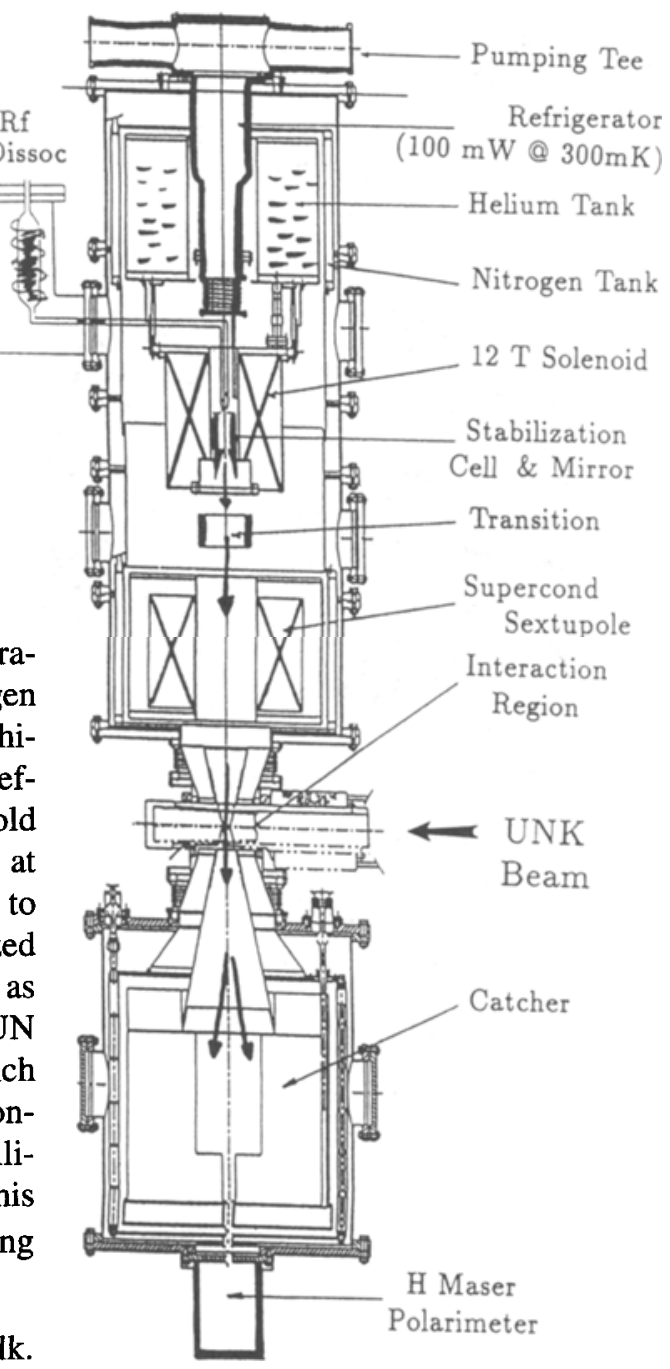

FIGURE 30. Michigan Mark-II Ultra-Cold Spin-Polarized $H$ Jet. thank the three secretaries of these two Symposia: Sharon Herzel, Janet Meadows, and Sandy Smith. They were enormously helpful and tolerant of the strange behavior of about 340 physicists; we physicists sometimes do good research but can be a problem to deal with personally. 
Figure 31 demonstrates an interesting situation where five different Committees have been organizing the two Symposia that are running together here in the Indiana Memorial Union. I have never before been to a meeting where there were five Committees. Two nights ago, I pointed out to Dr. Happer, who formerly directed research for DoE, that apparently DoE and NSF are now supporting advanced R\&D in Political Science. It was certainly interesting to watch the five Committees interact with each other.

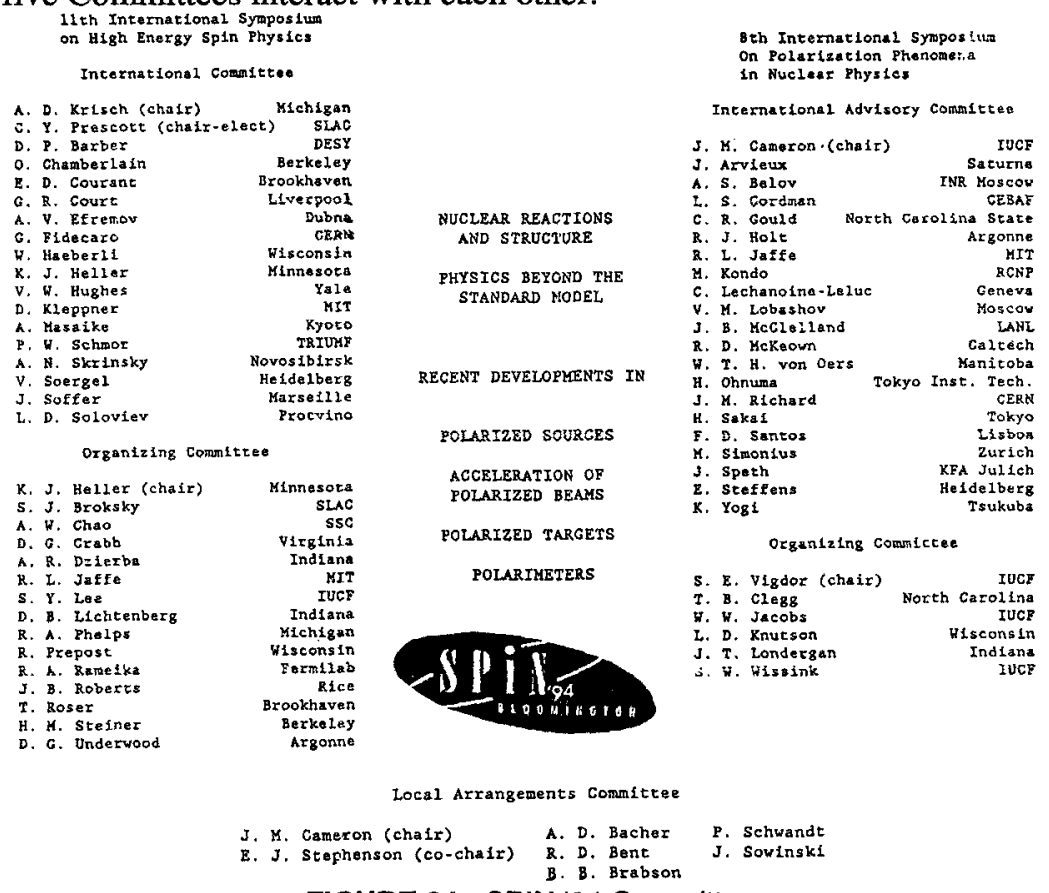

FIGURE 31. SPIN '94 Committees.

I would especially like to praise Ed Stephenson, the Co-Chair of the Local Arrangements Committee. To appreciate his contributions, let me briefly describe a typical meeting of the Chairs of these five Committees; the meeting would usually take place in a small conference room in the IUCF building. Typically, Professor Cameron and I would be rolling around on the floor, shouting at each other politically incorrect insults about each other's energy preference. Professors Vigdor and Heller would be sitting there looking mildly embarrassed, but nevertheless cheering us on a bit. Dr. Stephenson would ignore the insults and the noise and continue working on a list of candidates for the SOROS Foundation; he just kept doing his job. We should all thank Ed Stephenson; perhaps we should make him the Permanent Chair of the Local Arrangements Committee.

Next, I would like to announce some results of yesterday's Meeting of the International Committee for High Energy Spin Physics; the Committee Members 
are listed in Figure 31. The first announcement is that the 12th International High Energy Spin Physics Symposium will be held during 9-14 September 1996 in Amsterdam. Professor C.W. de Jager will be the Chair of the Organizing Committee; I am looking forward to a very exciting Symposium.

Next we approved the 5th Workshop on Polarized Ion Sources and Gas Targets. It will occur during 6-9 June 1995 in Cologne. Professor H.P.g. Schieck will chair the Workshop; anyone interested in participating should contact him.

We also approved the 6th Protvino Spin Workshop; it will take place during 18-23 September 1995 in Protvino. Professor S.B. Nurushev will again chair this Workshop; anyone interested in attending should contact him.

The 8th Workshop on Solid Polarized Targets was also approved. The exact date is not yet fixed, but it will be at TRIUMF in either May or June 1996; the Chair will be Professor P. Delheij.

We also had some discussions about the 13th and 14th High Energy Spin Physics Symposia, which will occur in the years 1998 and 2000. We have a tentative proposal from Prague for the year 2000; we certainly took no action on what could happen in the year 2000 , but it might be nice sometime to have the Symposium in Prague.

We talked informally about many places for the 1998 Symposium. It has been a long time since the Symposium was at one of the US national labs; it was at Brookhaven in 1982 and at Argonne in 1974, 1976, and 1978. Now SLAC, Fermilab, and Brookhaven may each have some interest. Argonne might also be interested; they no longer have an accelerator, but we should sometime return to Argonne where this Symposium series started. There was an informal proposal that the Symposium might be in Hawaii, jointly sponsored by America and Japan. This seems a bit complicated, as we again might need five Committees, but who knows? The 1998 Symposium could also be at some North American university that is heavily involved in High Energy Spin Physics. We have had very good experiences here and at Minnesota; I would note that Wisconsin is a very nice place. Another place we discussed was Lake Louise, Canada, where the 1986 Intersections Meeting occurred. One reason for thinking of Lake Louise is that we were all so pleased with the logistics here at Indiana; having the meeting rooms and many hotel rooms together in one building in a small town seems very good. Perhaps we should try to set this as a goal for the future. Lake Louise would be a change from the past in some ways, but we can think about new things. If anyone has any input on this, please send it to the Committee.

My next comment is about the International Committee Members. Four very distinguished Committee Members are retiring at the end of this year: Owen Chamberlain, Vernon Hughes, Daniel Kleppner, and Akiro Masaike. I would like to thank these four distinguished gentlemen for their long service. I am pleased to 
announce that there are four distinguished new Committee Members: John Cameron, Hiroyasu Ejiri, William Happer, and Yoshi Mori. These changes represent an effort to make the Committee even more international by increasing the Japanese representation. We also added two intermediate energy physicists: Professor Cameron and Professor Ejiri; we hope that this will encourage more intermediate energy and nuclear physicists to participate in the Amsterdam High Energy Spin Physics Symposium in 1996. By inviting more distinguished members of the Nuclear Polarization community to join our Committee, we hope to give a positive message that we are open to change. We look forward to many intermediate energy and nuclear physicists attending the Amsterdam meeting.

The trial partial merger of the two Spin Symposia was very exciting and exhausting but also very successful; we had a marvelous scientific meeting. I hope that John Cameron and I are still good friends, but the great success of the Spin '94 Meeting is what is really important.

Finally, this is my last Symposium as Chair; it has been an exciting, rewarding, and exhausting 20-year term. Our new Chair will be Charles Prescott of SLAC. I wish good luck to Charlie and all the rest of you.

\section{ACKNOWLEDGMENTS}

This work was partially supported by a Research Grant from the U.S. Department of Energy. I would like to thank Drs. A.M.T. Lin and L.G. Ratner and Ms. D.C. Barron for carefully proofreading this manuscript.

\section{REFERENCES}

1. I.M. Ternov, these Proceedings; A.A. Sokolov and I.M. Ternov, Sov. Phys. Dokl. 8, 1203 (1964).

2. A.D. Krisch, Z. Phys. C46, S133 (1990).

3. E.A. Crosbie et al., Phys. Rev. D23, 600 (1981).

4. T.J. Devlin and L.G. Pondrom, these Proceedings.

5. A. Yokosawa, Proc. of 10 th Int. Symp. on High Energy Spin Physics, eds. T. Hasegawa et al., p. 93 (Univ. Acc. Press, Inc. Tokyo 1993).

6. D.G. Crabb et al., Phys. Rev. Letters 65, 3241 (1990).

7. A. Thomas, R. Voss, R. Windmolders, D. Day, H. Jackson, J. Soffer and M. Anselmino, these Proceedings.

8. Workshop on Polarized Ion Sources and Polarized Gas Targets, L.W. Anderson and W.

Haeberli eds., AIP Conf. Proc. 293 (AIP, New York, 1993).

9. Y. Mori, these Proceedings.

10. Spin ' 93 Proceedings, S.B. Nurushev et al. eds., (IHEP, Protvino 1994).

11. Bad Honneff Workshop Proceedings, hopefully to be published; W. Meyer, these Proceedings.

12. M. Placidi, these Proceedings.

13. G. Voss, D. Barber, and H. Jackson, these Proceedings. 
14. V. Soergel, Unpublished comment at plenary session of 9th Int. Symp. on High Energy Spin Physics, Bonn 1990.

15. M. Woods and H.B. Steiner, these Proceedings.

16. Ya.S. Derbenev and A.M. Kondratenko, Part. Accel 8, 115 (1978).

17. A.D. Krisch et al., Phys. Rev. Letters 63, 1137 (1989).

18. B.B. Blinov et al., Phys. Rev. Letters 73, 1621 (1994).

19. R.A. Phelps et al., Phys. Rev. Letters 72, 1479 (1993).

20. D.D. Caussyn et al., Phys Rev. Letters 73, 2857 (1994).

21. S.E. Vigdor, these Proceedings.

22. H. Huang and T. Roser, these Proceedings; H. Huang et al., Phys. Rev. Letters 73, 2982 (1994).

23. F.Z. Khiari et al., Phys. Rev. D39, 45 (1989).

24. Y. Makdisi, these Proceedings.

25. Polarized Tevatron Progress Report, unpublished University of Michigan Report UM HE 94-15 (August 1, 1994).

26. CERN Courier, June 1994, pp. 18-20.

27. V.G. Luppov et al., Phys. Rev. Letters 71, 2405 (1993). 